\title{
Report on the GC-MBS Method for Correcting NaI Spectra for Transmission Loss in Hand-Held Instruments
}

October 8, 1997

Principle Investigator: Mohini Rawool-Sullivan

Contributing Researchers: Robert Estep and David Miko

Advanced Nuclear Technology Group (NIS-6)

Los Alamos National Laboratory

This work was sponsored by NBC LDRD, FY97

Sara C. Scott, project manager

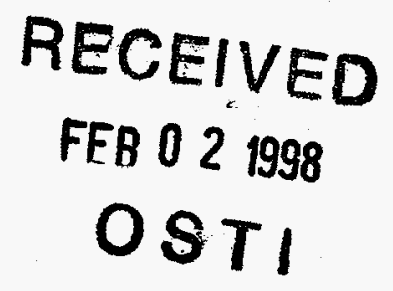

\section{PROJECT SUMMARY}

The goals of this project were (1) to develop a capability to study the scattered components in the NaI spectra of attenuated sources and (2) to evaluate the effectiveness of the gross count material basis set (GC-MBS) method in quantifying transmission losses from the shapes of measured Nal spectra. These goals are related, as the GC-MBS method involves a linear log-spectrum decomposition into MBS component spectra, and scattered gamma rays represent a significant nonlinear inteference. Eventually, we hope to understand the effect of the scattered components on the MBS decomposition and to develop ways to correct for inaccuracies. As of this writing we have not reached that longterm objective, so the two halves of this project are treated here as separate topics, with a separate section for each.

We have substantially achieved both of our project goals and are collecting additional data for two publications at the upcoming IEEE conference in Albuquerque, NM - one paper about our work on scattering and another on the GC-MBS method. This project report will contain preliminary portions of those two papers.

\section{Summary of Scattering Work}

We have developed a computer modeling capability using the Stanford University EGS4 simulation software. The model has been verified by simulating the spectrum of a ${ }^{137} \mathrm{Cs}$ source attenuated by different materials, by the generation of 11 -degree and 33degree scatter spectra from ${ }^{137} \mathrm{Cs}$, and by other tests. Experimentally measured spectra were found to be in good agreement with the simulations. A problem we encountered is that the simulations are very time consuming. In future work we will segment the simulations into managable subunits to speed the simulations and allow more complicated geometries to be modelled. Section I in this report describes our work on EGS4 simulation software. Section II describes experimental measurements of scatter components.

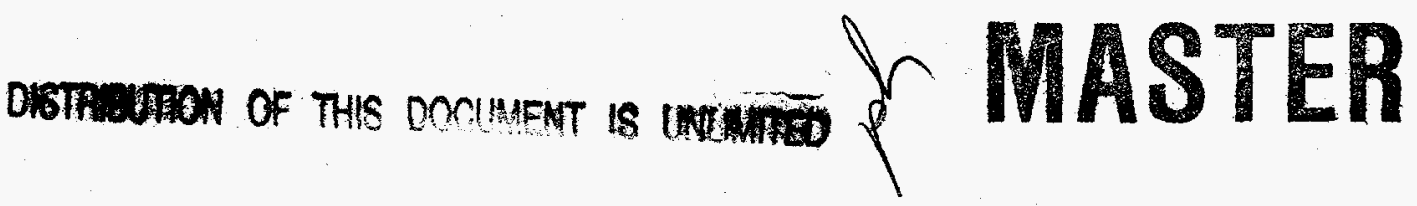




\section{DISCLAIMER}

This report was prepared as an account of work sponsored by an agency of the United States Government. Neither the United States Government not any agency thereof, nor any of their employees, makes any warranty, express or implied, or assumes any legal liability or responsibility for the accuracy, completeness, or usefulness of any information, apparatus, product, or process disclosed, or represents that its use would not infringe privately owned rights. Reference herein to any specific commercial product, process, or service by trade name, trademark, manufacturer, or otherwise does not necessarily constitute or imply its endorsement, recommendation, or favoring by the United States Government or any agency thereof. The views and opinions of authors expressed herein do not necessarily state or reflect those of the United States Government or any agency thereof. 


\section{DISCLAIMER}

Portions of this document may be illegible electronic image products. Images are produced from the best available original document. 
To help understand the effect of potential factors affecting accurate determination of the source strength

$\because$ calculations using GC-MBS method, we have developed a capability within NIS-6. Mote-Carlo calculations are useful in this regard as one can control the various parameters involved in given set-up. We obtain the electron gamma shower code from SLAC. The Electron Gamma Shower 4 (latest version) or EGS4 system of codes is a general purpose package for the Monte Carlo simulation of the coupled transport of electrons/positrons and photons in an arbitrary geometry for particles with energies above a few $\mathrm{keV}$ up to several TeV. The geometry is written by the user to simulate his or her experimental set-up. In addition to this user is also responsible for keeping the track of quantities of interest and coding the input source of gamma rays, electrons or positrons. The most difficult part was we had to learn MORTRAN language for the best use of this code. Using this code currently we are studying various factors which may affect our set-up. In next section we have described briefly the photon interaction with matter.

Background on the photon interaction

Main interactions of $\mathrm{x}$-rays and gamma rays in matter are photoelectric effect, Compton scattering and pair production. These three interactions give two qualitative features to the $\mathrm{x}$-rays and gamma rays. These features are that these rays are far more penetrating in matter than charged particles and a beam of photon is not degraded in energy as it passes through a thick material but is only attenuated in intensity. The first feature comes from the fact that the three modes of interactions have much smaller cross-sections. The second feature results because all of the above mentioned processes remove photons completely from the beam thus reducing the number of photons from the beam. The photons which pass straight through are the photons which have not suffered any interaction at all and thus have their original energy. In following sections we discuss the above mentioned photon interactions in some detail.

\section{Photoelectric effect}

This effect involves the absorption of photon by atomic electron resulting in the ejection of the electron from the atom and the permanent loss of the photon. The photoelectric cross-section depends on the energy of the photon, however this dependence goes as $\mathrm{Z}$ to the $4^{\text {th }}$ power or $5^{\text {th }}$ power. Thus photoelectric crosssection is larger for high $\mathrm{Z}$ material. For a given material this interaction is more probable at lower energies than at higher energies.

\section{Compton interaction}

This is the best understood photon interaction. This is the scattering of photons from free electrons. In matter electrons are bound with energies of the order of eV. Thus to a photon with energy of the order of a $\mathrm{keV}$ this electron is essentially free. The total probability per electron for the Compton scattering to occur is calculated from Klein-Nishima formula and is given by

$$
\sigma_{c}=2 \pi r_{e}^{2}\left\{\frac{1+\gamma}{\gamma^{2}}\left[\frac{2(1+\gamma)-1}{1+2 \gamma} \ln (1+2 \gamma)\right]+\frac{1}{2 \gamma} \ln (1+2 \gamma)-\frac{(1+3 \gamma)}{(1+2 \gamma)^{2}}\right\}
$$

where $\gamma=h v / m_{e} c^{2}$. This cross-section can be divided into two parts the Compton scattered and Compton absorption cross-section. Compton scattering results in the scattered photon and the knocked out electron. Since the electron is stopped by the material, this is the average energy fraction absorbed by the material in Compton scattering. The probability $(\mathrm{P})$ that the Compton scattering will occur per unit distance ( $\rho \mathrm{dX}$ in units of mass per unit area e.g. $\left.\mathrm{mg} / \mathrm{cm}^{2}\right)$ in a given material is then $\mathrm{P} /(\rho \mathrm{dX})=\left(\mathrm{N}_{\mathrm{A}} / \mathrm{A}\right) * \mathrm{Z} * \sigma_{\mathrm{C}}$ where $\mathrm{N}_{\mathrm{A}}$ is the Avagadro's number. If we note here that for most materials except hydrogen $A \approx 2 \mathrm{Z}$ to $A \approx 2.6 Z$ then we find that quantity $P /(\rho d X)$ is almost independent of $Z$.

\section{Pair production}


scattering per unit distance (in units such as $\mathrm{cm}$ ) is dependant on the density of the material and increases with this density. This $\mathrm{NaI}$ spectra results from following set of photons and electrons:

، 1. The set of photons which passed through the absorbers without any interaction in the absorber but which did have interaction in $\mathrm{NaI}$.

2. The set of photons which had Compton interaction in the absorber and then they had another interaction in $\mathrm{NaI}$.

3. The set of photons which undergo Rayleigh or Thomson scattering and then have interaction in the NaI.

You can also see in this figure that the valley between the Compton and the photopeak is shallower as the denser absorbers are used. These events are made up of mainly the photons which undergo Compton scattering (and maybe to some extent Thomson or Rayleigh scattering) in the absorber where outgoing photons have somewhat less energy and then these photons had photoelectric interaction in the NaI crystal. If this is true then for given material we should observe shallower valleys between the Compton edge and the photopeak with increased thickness.

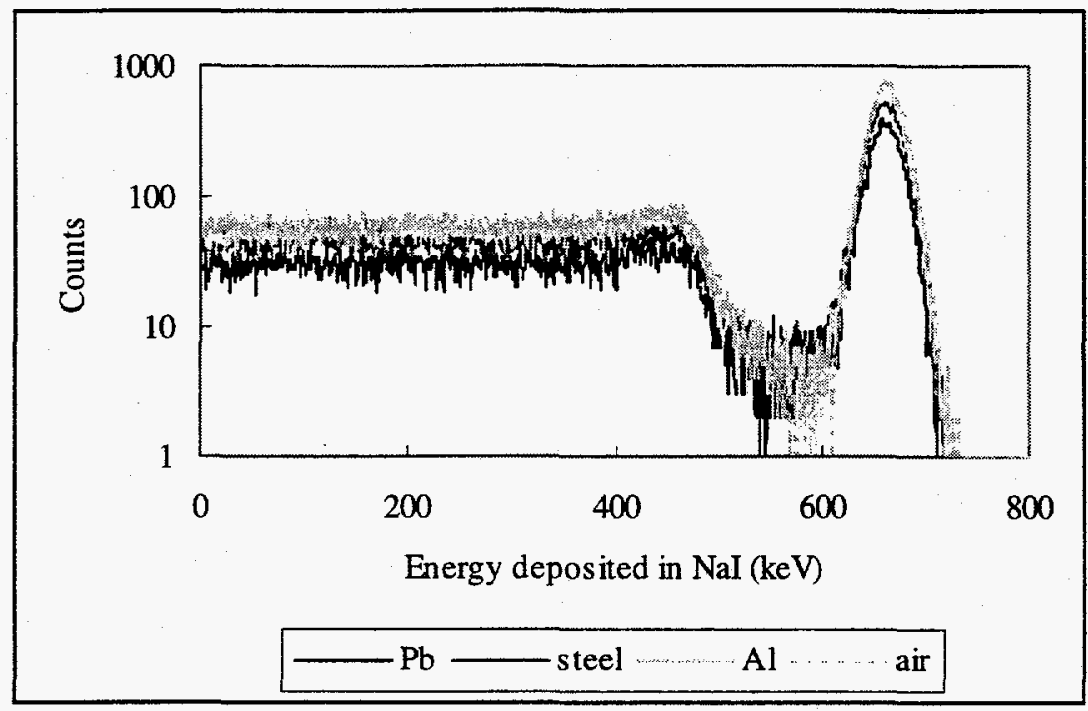

Figure 2: Cs-137 spectra attenuated through various absorbers

Case 2: Different thickness

Here experimental setup was exactly the same as previous case but the aluminum absorber thickness is varied. As you can see from the figure that the photopeak decreases in the amplitude as a function of the increased thickness as more photons suffer interaction in the absorber with increased thickness. The valley is shallower as predicted in the previous case. 
II. Compton Side-Scatter Effects on Sodium Iodide Detector

\section{Side-Scatter Experiment Overview}

A series of experiments were performed to determine the contribution of Compton sidescatter on the gamma spectrum of a sodium-iodide detector. Scatter spectra were determined for aluminum, steel and lead over a range of energies. The quantification of this contribution should enable better source strength resolution using gross-counts techniques by isolating the scattering material from the attenuating material.

A 2" diameter NaI detector was used for the measurements. The source was placed 20 " from the detector along the detector axis. To minimize scatter, the various experimental pieces were raised several inches off the table. The scattering and transmission pieces were placed at the midpoint of the source-detector axis. Several circular plugs, 2" in diameter, were constructed. These were made of aluminum, polyethylene, and lead. These were used to measure the transmission spectra. This was done so that this spectrum could be subtracted from the scatter spectrum to yield the scatter component. A fourth plug (of the same diameter) was made of 2" thick tungsten to block all the peak transmission. By eliminating the peak, the Compton scatter component could be more easily distinguished from the rest of the spectrum.

Scattering pieces were constructed of aluminum, stainless steel, and lead. For the aluminum and steel, two sizes of scattering rings were constructed. The first was a half-ring $\left(180^{\circ}\right)$ with a 1 " inner radius and a 2 " outer radius, 1 " thick. This piece was made to fit over the attenuating plug. The second scattering piece was another half-ring with a 2" inner radius and a 3 " outer radius, 1" thick. This piece fit over the first scattering ring. The lead scattering pieces were half rings of 1 " inner radius and 3" outer radius, of differing thicknesses.

Since the scattering materials were all concentric along the source-detector axis, scattering angles were easily discernible. The scattering angle covered by the plug was $0^{\circ}-11^{\circ}$. The inner ring covered roughly $11^{\circ}-22^{\circ}$. The outer ring covered $22^{\circ}-33^{\circ}$. Therefore, the lead ring covered scattering angles of $11^{\circ}-33^{\circ}$. These three scattering ranges will be referred to as $17^{\circ}, 28^{\circ}$, and $22^{\circ}$, respectively.

There are three main factors in the determination of Compton scatter: source, distance, and attenuation. Compton scatter is the scatter of a photon off of a "free" electron (binding energy is considered to be negligible). Therefore, the type of scattering material is largely irrelevant to the production of Compton scatter. The electron density is the important factor. There is a strong angular and energy dependence that will be examined in the next section. Since the geometric efficiency decreases as the inverse square of the distance, the farther the scatterer is from the source-detector axis, the lower the count rate. Although the type of scattering material is irrelevant in the production of Compton scatter, it is paramount in the self-absorption of the Compton photons. For example, low energy photons produced in lead likely will not escape. 


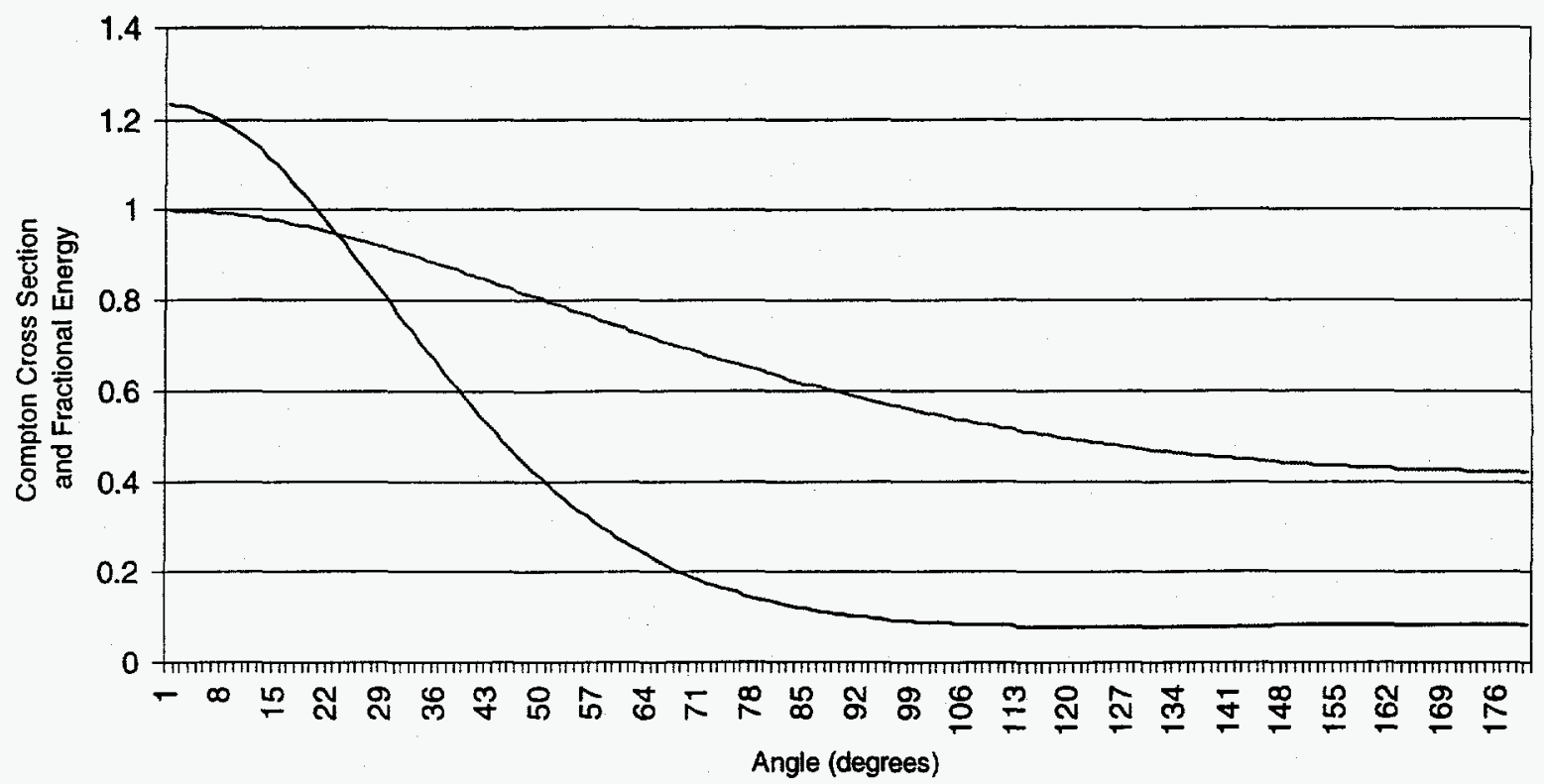

Figure 2: Compton scatter cross section (normalized per electron) and the fractional energy of the scattered photon. Initial photon energy is $350 \mathrm{keV}$.

Compton Scatter Cross Section and Fractional Scatter Energy for 661 keV Photons

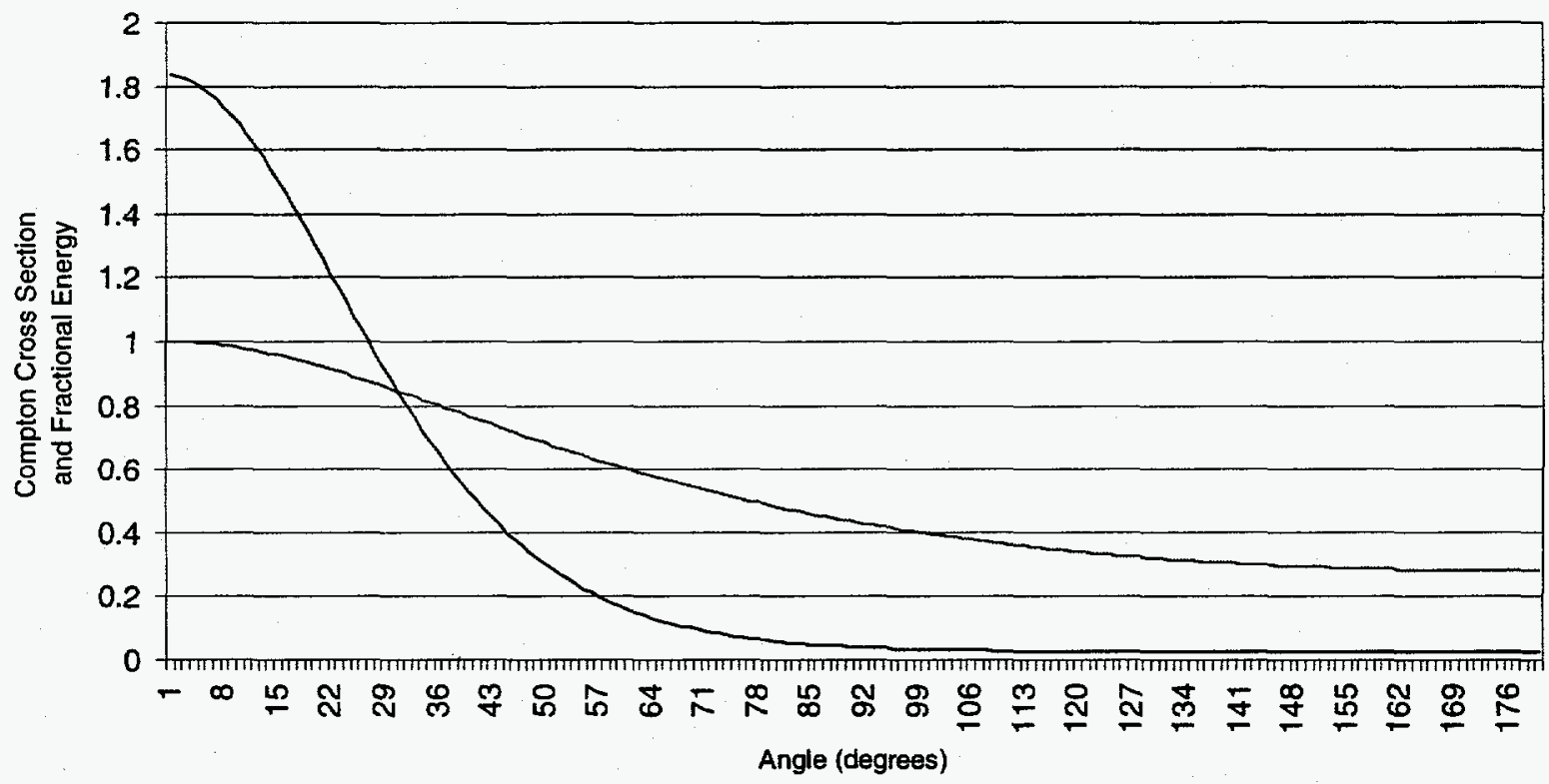

Figure 3: Compton scatter cross section (normalized per electron) and the fractional energy of the scattered photon. Initial photon energy is $661 \mathrm{keV}$. 
The spectra taken with tungsten (2" thick), results in the total loss of the peaks. Having such a thick layer of absorber is not a realistic scenario. However, it is effective in reducing the . background counts. In this way, a "cleaner" scatter spectrum is acquired since the scatter/background ratio is significantly increased.

\section{$\underline{\text { Scatter Spectra }}$}

Using the experimental configuration previously described, spectra were measured with the various attenuating materials along with the scattering materials. For the aluminum and steel rings, there were two sets of rings. Therefore, scatter spectra from 1" and 2" thicknesses were measured. For the lead rings, pieces of varying thickness were made. The total thickness for the lead scattering rings was approximately $5 / 16$ ".

Figures $8 \mathrm{a}-\mathrm{b}$ show the scatter spectra from the cesium source. The scattering material is steel. The 2" tungsten plug is used to block the transmission source. Figure 8a gives the spectrum off of the inner $\left(17^{\circ}\right.$ scatter) ring. Figure $8 \mathrm{~b}$ gives the spectrum off of the outer $\left(28^{\circ}\right.$ scatter) ring. The shift in the peaks is consistent with that predicted by Figure 4 for an initial photon energy of $661 \mathrm{keV}$.

For the cesium source, there was little attenuation of the scattered photons in 1" of steel. The scatter spectrum from 2" of steel was comparable to 1". Therefore, the added scatter source was compensated by a loss of photons in the extra material.

Figures $9 \mathrm{a}-\mathrm{b}$ show the scatter spectrum from the barium source. The scattering material is aluminum. In order to get a "cleaner" scatter spectrum, the tungsten plug is used to attenuate the transmission. 1" scattering rings are used as the scatter source. Figure 9a gives the spectrum off of the inner $\left(17^{\circ}\right.$ scatter $)$ ring. Figure $9 \mathrm{~b}$ gives the spectrum off of the outer $\left(28^{\circ} \mathrm{scatter}\right)$ ring.

The scatter spectra from the steel and lead were significantly smaller than for aluminum (with the barium source). This is due to the self-absorption of the scattered photons in these materials. Using the 1" aluminum plug resulted in a very "noisy" spectrum.

Figure 10 shows the scatter spectrum from the plutonium source. The scattering and transmission material is $1 / 8$ " lead. Thicker layers resulted in self-absorption blocking the scattered photons. This is a good example of a "noisy" spectrum.

\section{Discussion of Scatter Spectra}

In experimental measurements, the statistical uncertainty for the counts in a given channel is the square root of the counts. When determining the scatter component, a transmission spectrum is subtracted from a scatter spectrum. Therefore, the uncertainty for a channel of the scatter component is the sum of the square roots of scatter and transmission spectra. If the scatter component is small compared to the transmission, then the resulting uncertainty will be large, and the spectrum will look like that of Figure 10. 


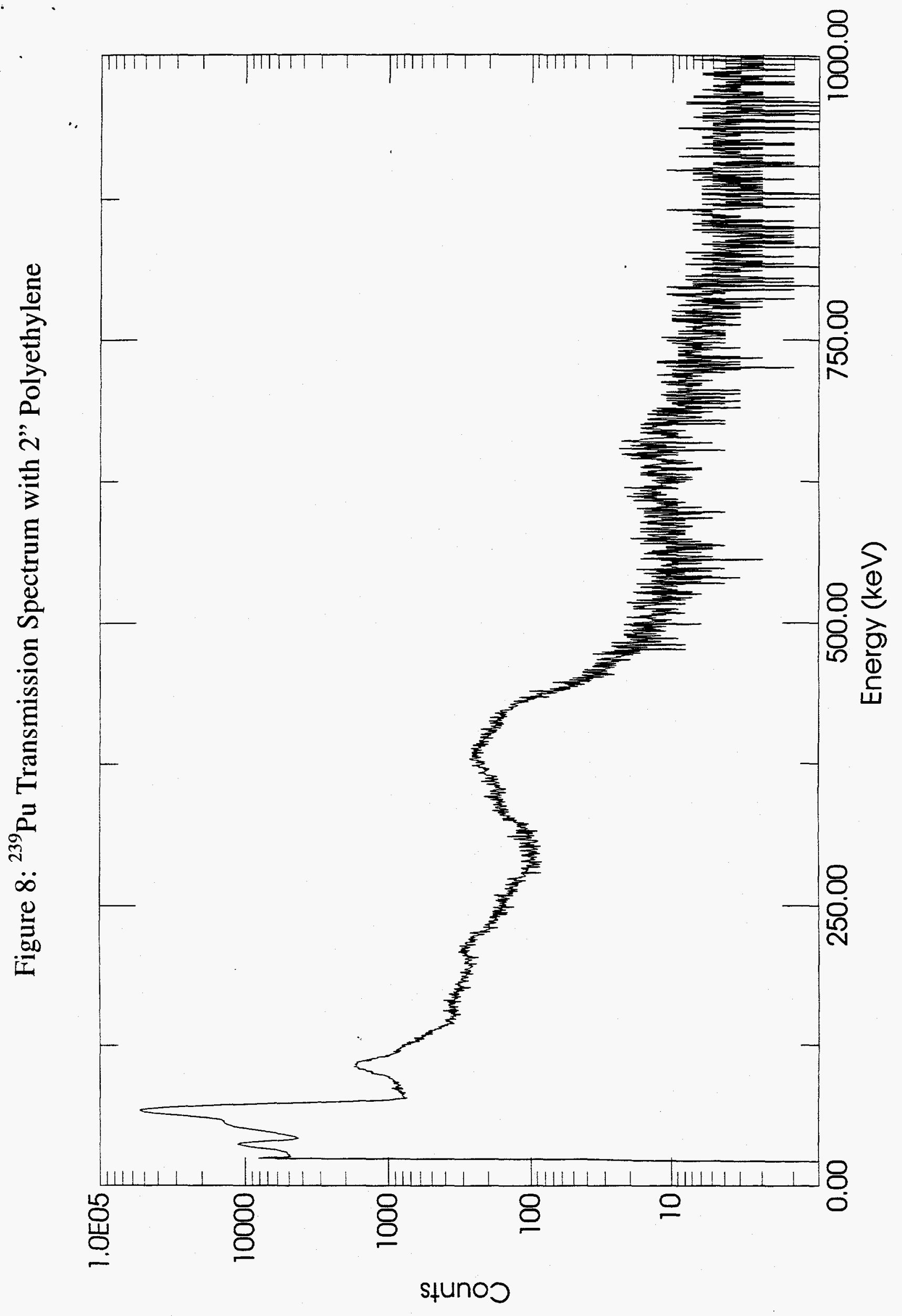




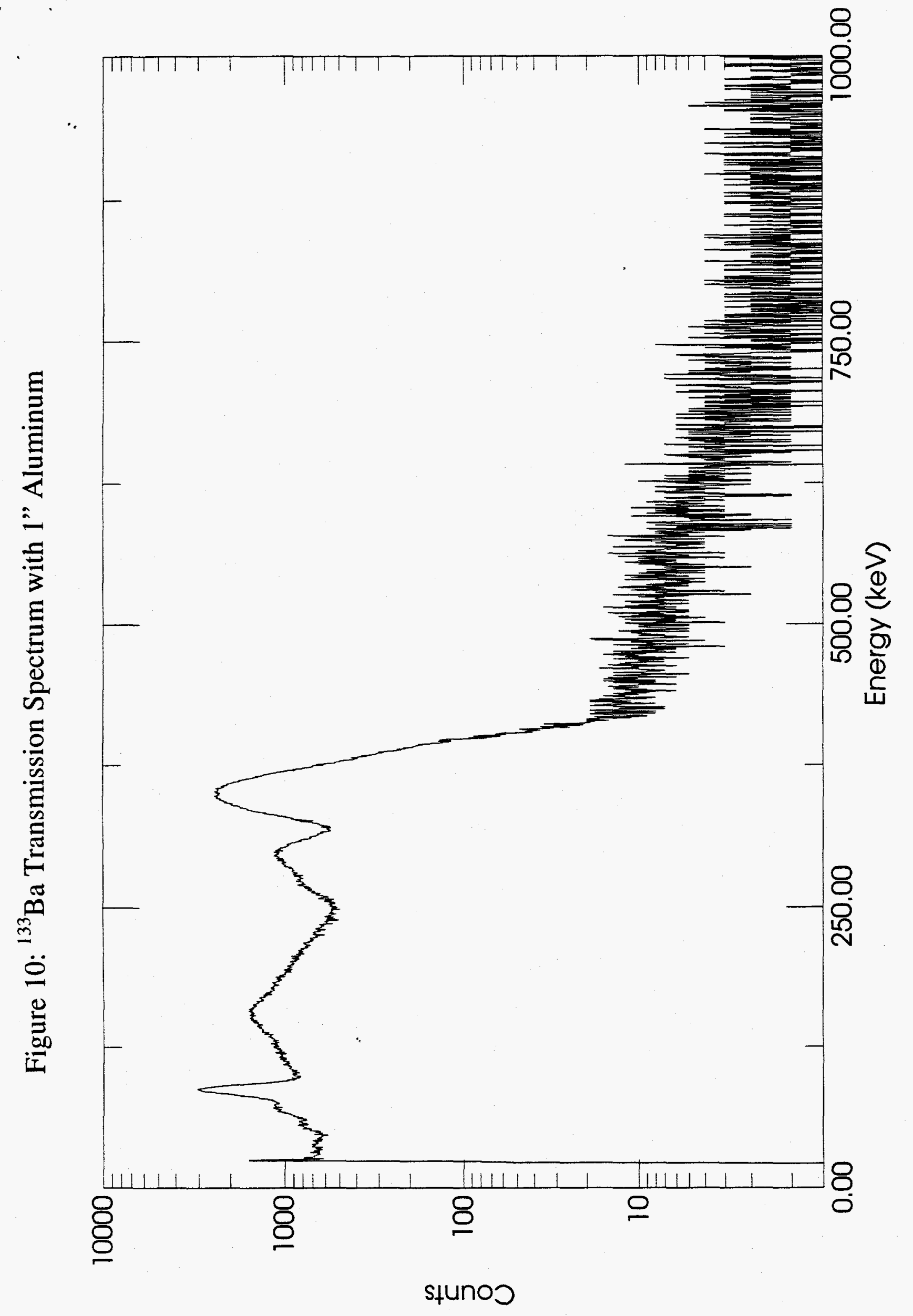




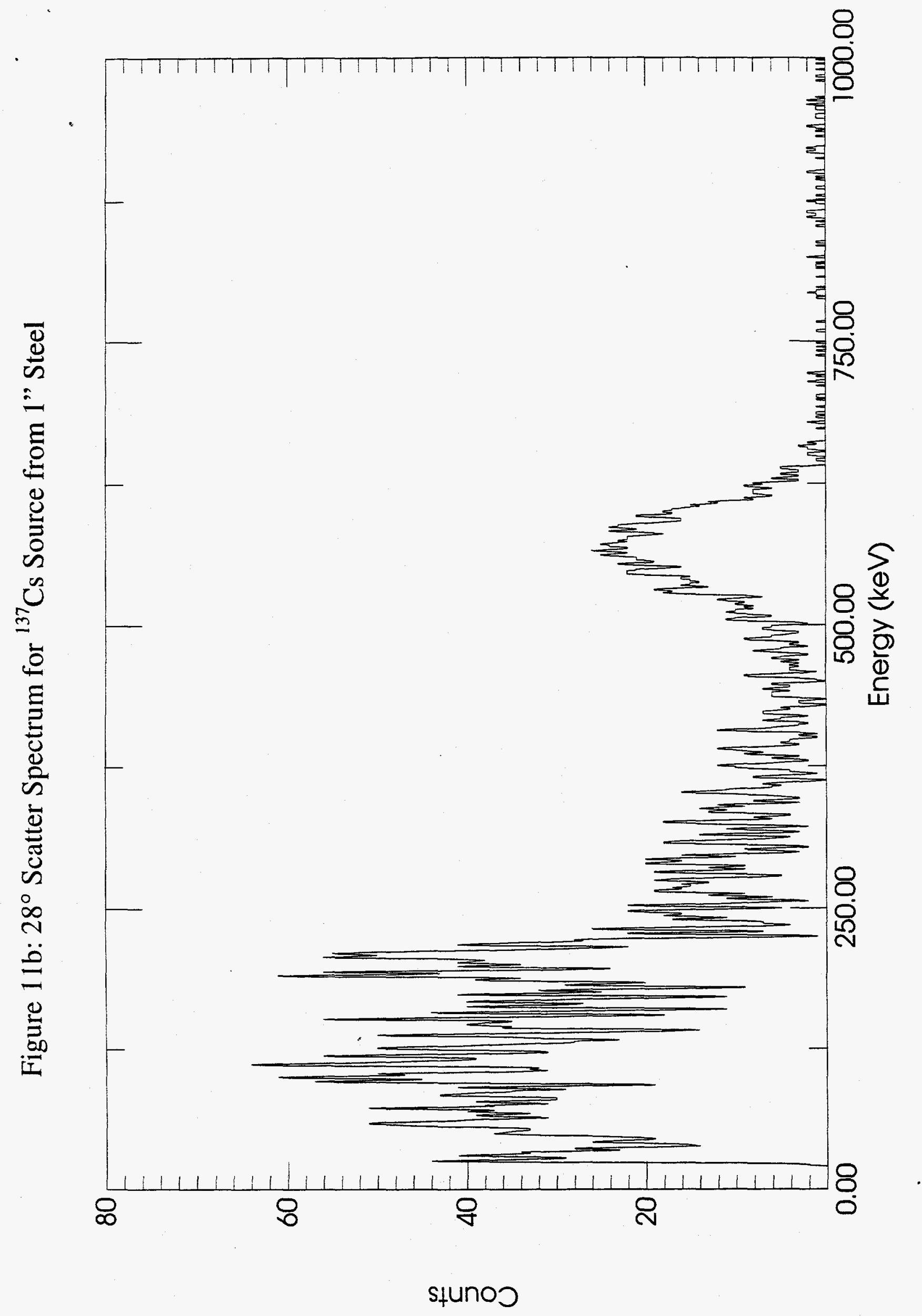




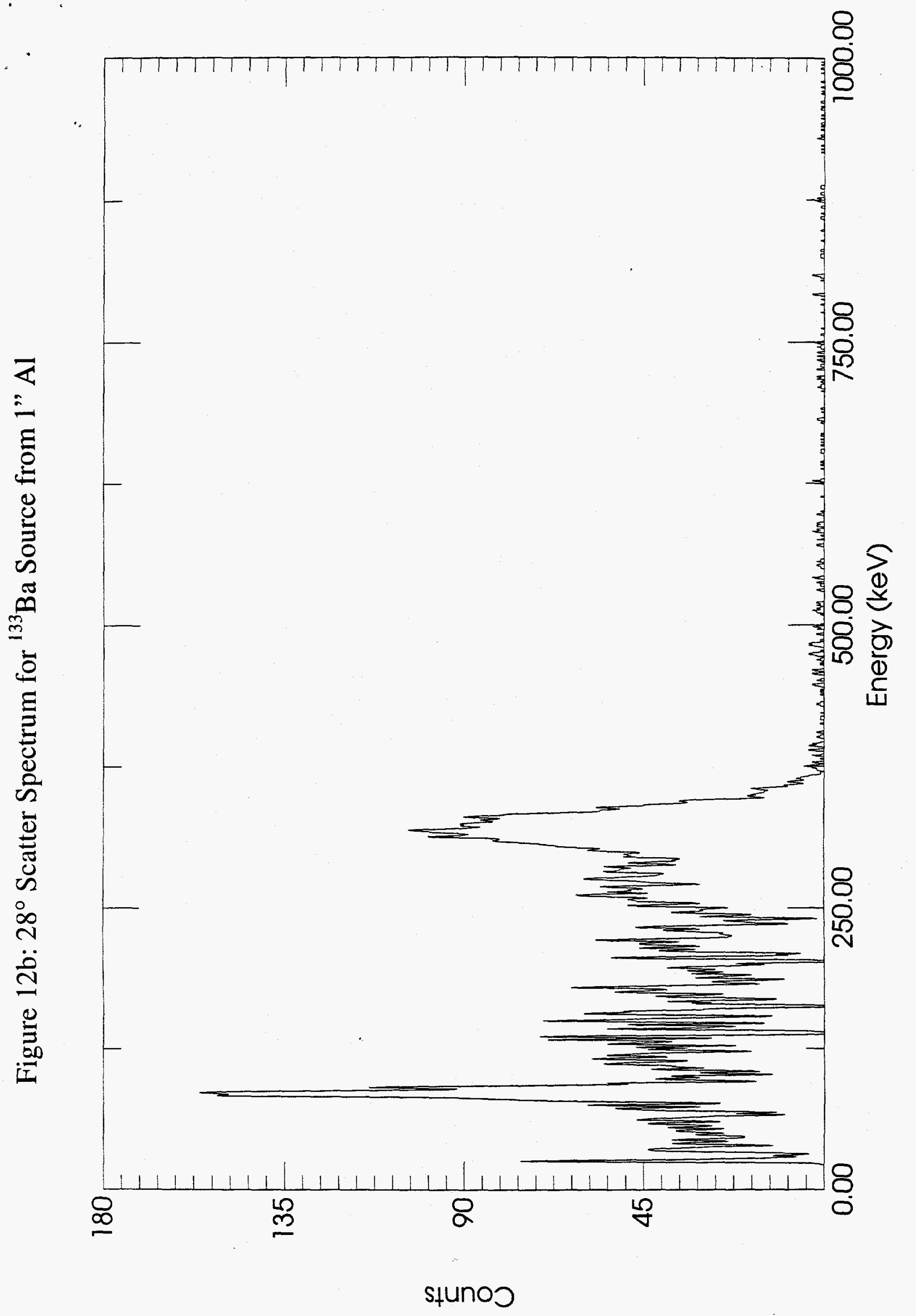




\section{GC-MBS METHOD DEMONSTRATION}

The GC-MBS method ${ }^{1}$ uses the distortion in the unknown's spectrum shape to estimate the attenuation loss in intervening absorbers when the isotopic composition of the source is known. In essence, the source spectrum is restored to its unattenuated shape and amplitude. The distortion of the spectrum due to scattered gamma rays is treated approximately by including representative side-scatter basis components in the MBS, either as stand-alone scatter bases or as components of regular material bases.

The direct use of the GC-MBS method to obtain accurate source strength estimates should find numerous practical applications. The most important use, however, may be in improving the accuracy of isotopic composition measurements in situations where the $\mathrm{NaI}$ spectrum is distorted by a container or other intervening material. A problem of particular importance is measuring the enrichment of uranium blends. Because the enrichment measurement is based on the observed ratio of high- to low-energy gamma rays, and because the low-energy gamma rays are more strongly attenuated in passing through absorbers, the enrichment measurement must be calibrated separately for every different absorber that might be encountered. For example, an enrichment measurement calibrated for plastic bottles would not be valid for measurements on stainless steel pipes. By simultaneously solving for the enrichment and the tranmission loss, the enrichment measurement could be applied to arbitrary container types, greatly improving its usefulness.

\section{MATHEMATICAL DETAILS}

We apply the GC-MBS method to NaI spectra by treating each channel as if it were an independent full-energy peak. No continuum subtraction is performed; hence the term "gross count." To estimate transmission loss we compute effective attenuation coefficients for each channel from reference transmission spectra measured through a set of one or more basis materials, rather than using the known or calculated attenuation coefficients for gamma rays at the corresponding channel energies. Because we are working with entire spectra, we refer to the GC-MBS analysis as a spectrum decomposition or a response-function fit. In fact, the MBS part of the analysis is the same as would be used for individual continuum-subtracted gamma-ray peaks measured with a germanium detector.

The remainder of this section gives details of the GC-MBS method as applied in emission-only situations.

\section{The GC-MBS model without scatter components}

Let $\mathrm{X}$ be the isotope or mixture of isotopes to which the method will be applied (e.g., $\mathrm{Pu}, \mathrm{HEU}$, etc). We measure an $\mathrm{N}$-channel spectrum $\mathbf{c}$ from an unknown mass (mass) of $\mathrm{X}$ at a distance $r$ from the detector for a count time $t$, attenuated by unknown amounts 
With an absorber composition represented by $\rho_{\mathrm{Pb}}$ and $\rho_{\mathrm{Al}}$ (where $\rho_{z}$ is the MBS partial density of material Z), we can write

$$
f_{\mathrm{i}}=k \cdot \exp \left\{-\rho_{\mathrm{Pb}} \cdot \mathrm{b}_{\mathrm{Pb}, \mathrm{i}}-\rho_{\mathrm{Al}} \cdot \mathrm{b}_{\mathrm{Al}, \mathrm{i}}\right\},
$$

which leads to

$$
\begin{aligned}
& b_{i}=-\ln \left(f_{i}\right)=\rho_{P b} \cdot b_{P b, i}+\rho_{A l} \cdot b_{A l, i}-\ln (k), \text { or } \\
& b_{i}=\rho_{P b} \cdot b_{P b, i}+\rho_{A A} \cdot b_{A l, i}+x,
\end{aligned}
$$

where

$$
x=-\ln (k) \quad .
$$

Note that the exponential term in (6) is the MBS formulation of Beer's law for the attenuation of gamma rays and predicts the transmission of the unknown source. ${ }^{2}$ The partial densities $\rho$ are the fractions of the basis material thicknesses that approximate the attenuating properties of the absorber as a function of energy. That is, a thickness $\rho_{\mathrm{Pb}} \cdot d_{\mathrm{Pb}}$ of $\mathrm{Pb}$ combined with a thickness $\rho_{\mathrm{Al}} \cdot d_{\mathrm{Al}}$ of $\mathrm{Al}$ approximates the absorber.

Eqn. (7) represents a linear system of $N$ equations in the three unknowns: $\rho_{\mathrm{Pb}}, \rho_{\mathrm{Al}}$, and $x$. This can be written in matrix form as

$$
\mathbf{b}=\mathrm{U} \cdot \boldsymbol{\rho},
$$

with the MBS solution vector $\rho=\left[\begin{array}{lll}\rho_{\mathrm{Pb}} & \rho_{\mathrm{Al}} & x\end{array}\right]^{T}$ and data vector $\mathbf{b}=\left[\begin{array}{lll}\mathrm{b}_{0} & b_{1} & \ldots . . b_{N-1}\end{array}\right]^{T}$. The transformation matrix $U$ has as its three columns the basis $N$-vectors $\mathbf{b}_{\mathrm{Pb}}, \mathbf{b}_{\mathrm{Al}}$, and 1 (a vector of all l's). That is,

$$
\mathrm{U}=\left[\begin{array}{ccc}
\left\lceil\mathrm{b}_{\mathrm{Pb}, 0}\right. & \mathrm{b}_{\mathrm{Al}, 0} & 1 \\
\mathrm{~b}_{\mathrm{Pb}, 1} & \mathrm{~b}_{\mathrm{Al}, 1} & 1 \\
\cdot & \cdot & \cdot \\
\cdot & \cdot & \cdot \\
\dot{\mathrm{b}_{\mathrm{pb}, N-1}} & \mathrm{~b}_{\mathrm{Al}, N-1} & \dot{1}
\end{array}\right]
$$

Solution of the system of equations (9) gives the mass almost directly as the constant term, $x$. For the general case where $r \neq r_{0}, t \neq t_{0}$, the mass is given by

$$
\text { mass }=\operatorname{mass}_{0} \cdot t_{0} \cdot r^{2} \cdot \exp \{-x\} /\left(t \cdot r_{0}{ }^{2}\right) \quad .
$$

The GC-MBS model with scatter components 
would add about $400 \mathrm{~g}$ to the weight of a hand-held instrument, was flush with the face of the detector and so did not act as a collimator. Data were collected with a PC compatible

computer running Ortec's Gamma-Vision (version 4.10) software and an Ortec model 918 multichannel analyzer card. All spectra had 4096 channels, with an approximate energy calibration of $0.5 \mathrm{keV}$ per channel. A small ${ }^{241} \mathrm{Am}$ source taped to the detector in a fixed position was used for gain shift corrections. The model 918 's slow conversion time of $\approx 75$ $\mu$ limited our ability to count at high rates, which insulated us to some degree from pileup and other high-rate effects. Count times in most cases were from two to five minutes. Background counts were typically ten to twenty minutes. The experimental area in which we performed our tests has a moderately high gamma background rate $(75-100 \mathrm{cps})$ due to its proximity to a radioactive source storage area.

The absorbers used in studying HEU spectrum attenuation are listed below in Table I. The absorbers were of various sizes, having been obtained from a pile of waste material in our group's machine shop. All the absorbers had a planar or slab shape. The "transmission" column gives the gross counts, after gain correction and subtraction of room background, in channels 210-500 of the transmitted HEU spectrum, relative to the unattenuated case (labelled "air"). This region-of-interest brackets the group of lowenergy peaks from uranium. The materials with asterisks were also used for depleted uranium transmission measurements.

We used a 100-g metallic HEU source for transmission measurements through all the absorbers listed, and a 10-kg depleted uranium source for measurements through a limited number of materials for the simulation of variable-enrichment measurements. The depleted $U$ source was counted at 9 inches from the detector. Rather than repeating all the transmission measurements for the HEU source at different distances, we made the nonbasis transmission measurements at 6.25 -inches from the detector and measured separate HEU basis spectra at 6.25 inches, 3.5 inches, and 9 inches from the detector to simulate measurements with a source-detector distance different from that used in measuring the reference spectra. This also simulates (approximately) different source strengths at the same position.

To simulate variable-enrichment uranium we summed HEU and DU transmission spectra in different ratios. Although it is unlikely that the spectra so obtained will exactly correspond to real mixtures of HEU and DU (because of self-attenuation differences related to the physical forms of these particular sources), this should be a reasonable test of the effects of varying isotopics on the GC-MBS results. 
distance. The squares represent data fitted using basis spectra taken at 6.25 inches; the diamonds used basis spectra taken at 3.5-inches; and the circles used basis spectra taken at . 9 inches. The results were essentially the same at the three distances, so we are confident that corrections are not being skewed toward the reference-spectra source strengths.

Figure 15 shows the our results for an GC-MBS analysis of the transmission of the 100-g HEU source through a set of carbon steel absorbers. Again, we have plotted the corrected versus uncorrected source strength. A one-material dual basis set of $1 / 4-$ and 3/4-inches of iron was used to fit the spectra. Restricting the range of materials this way improves the accuracy of the fit significantly, as was expected.

\section{Uranium Enrichment Measurements}

From a safeguards point-of-view the most important isotopes for handheld instrument applications are uranium and its blends, ranging from $\mathrm{HEU}\left(\geq 90 \%{ }^{235} \mathrm{U}\right)$ to depleted $\mathrm{U}\left(<0.72 \%{ }^{235} \mathrm{U},>99.3 \%{ }^{238} \mathrm{U}\right)$. The low-energy gamma rays in ${ }^{235} \mathrm{U}$ - ranging from 143 to $205 \mathrm{keV}$ - present a particular challenge for GC-MBS spectrum analysis because 1) at such low energies the gamma-ray attenuation coefficient varies widely with $\mathrm{Z}$ and with energy and because 2) spectrum distortions caused by low-angle scattering are most severe at lower energies. On the other hand, the $1001-\mathrm{keV}$ gamma ray in ${ }^{238} \mathrm{U}$ provides an easy and direct method for determing the enrichment of the uranium when other factors are constant. This is illustrated in figure 16, which compares the NaI spectra of unattenuated uranium blends varying from natural $(.72 \%)$ to enriched $(90 \%)^{4}$. As can be seen, the intensity of the low-energy peaks relative to the $1001-\mathrm{keV}$ peak and its associated continuum are directly related to the enrichment. Methods for determining enrichment can thus be as simple as taking the ratio of the counts below and above 250 $\mathrm{keV}$ (say) and matching it against a table of known values. A more sophisticated treatment would use spectrum decomposition (response function fitting) to determine the fractions of low- and high-enriched uranium in the sample.

The main problem encountered when measuring uranium isotopics as described above is that one must calibrate separately for every set of attenuating materials that will be between the source and the detector. If either the amount or type of intervening material is unknown, a potentially large inaccuracy is introduced that can render the technique unreliable. This is because the low energy gamma-ray group will be more attenuated than the high energy group in any material. For this reason, an important use for the GC-MBS method - to the extent it is successful - will be in allowing accurate uranium enrichment measurements to be made through arbitrary absorbers.

To illustrate the improvement in enrichment measurements that can be obtained by simultaneously fitting the transmission loss and the isotopic mix, we manually generated a "blended" data spectrum by summing in equal amounts transmission spectra from the 100$\mathrm{g}$ HEU source and a $2-\mathrm{kg}$ DU source. Nominally, this represents a blended source with a $5.4 \%{ }^{235} \mathrm{U}$ content, although self-attenuation considerations have not been accounted for, so it is not clear what actual blend is represented. The sources were both transmitted 


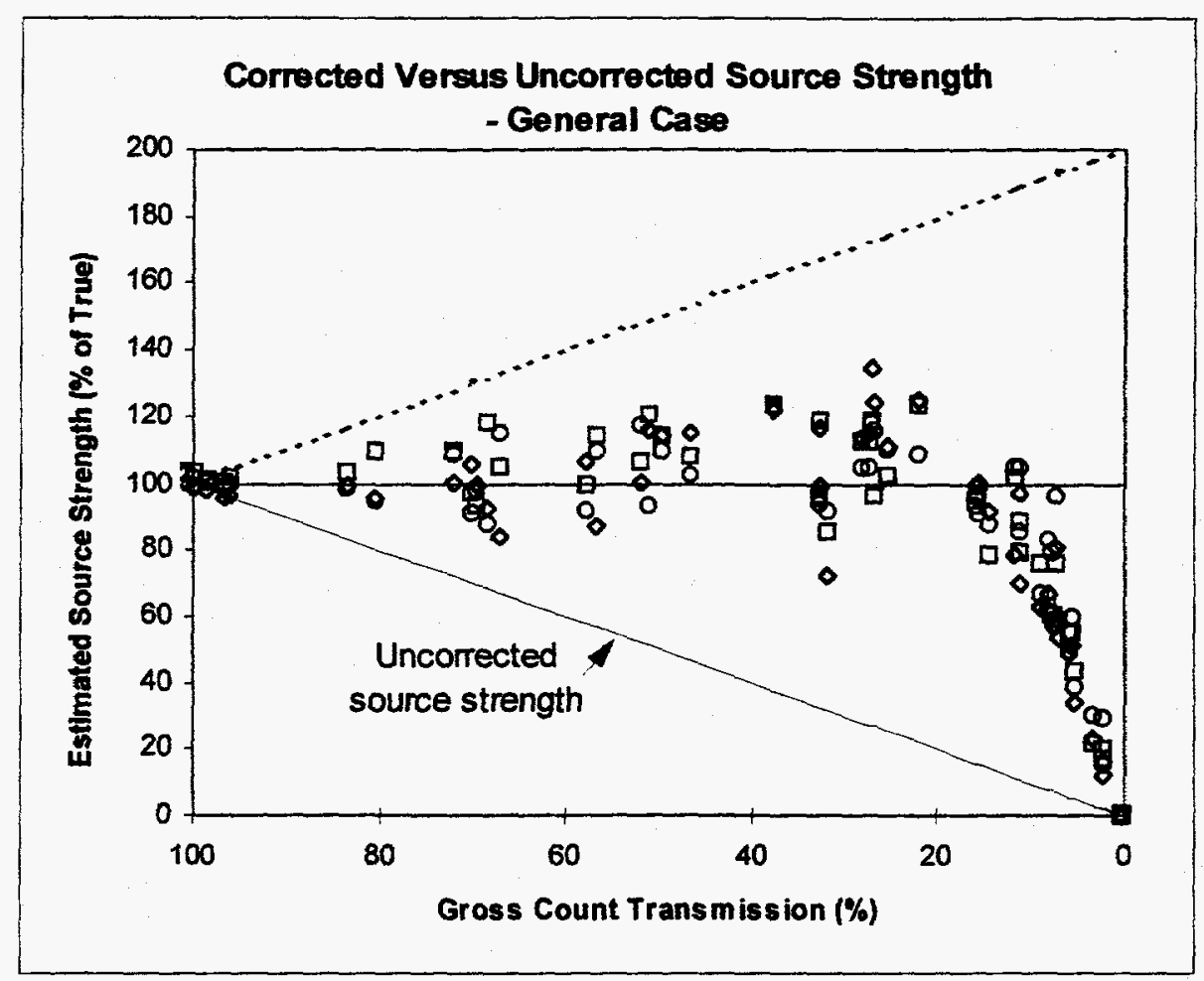

Figure 14. Corrected versus uncorrected source strength for transmission of a 100-g HEU source through the absorbers listed in Table I. A six-element dual basis set of .01- and .05inch $\mathrm{Pb}, 1 / 16$ - and 1/4-inch $\mathrm{Cd}$, and 3/8- and 15/8-inch Al was used to fit spectra attenuated by materials ranging from polyethylene to $\mathrm{Pb}$. Spectra were measured at a 6.25 -inch source-detector distance. The squares represent data fitted using basis spectra taken at 6.25 inches; the diamonds used basis spectra taken at 3.5-inches; and the circles used basis spectra taken at 9 inches. 


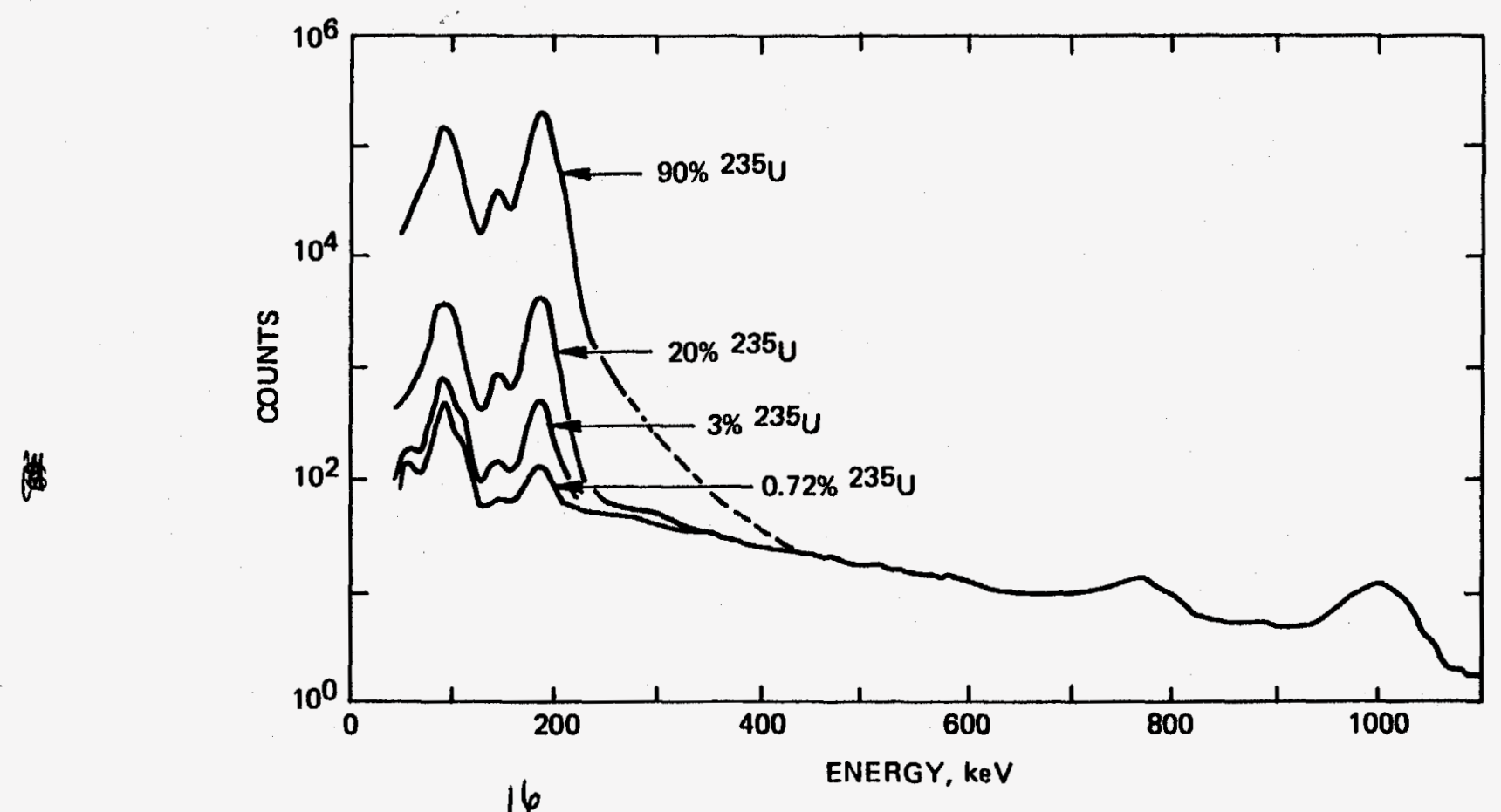

FIGURE - Gamma-ray spectra of natural uranium $\left(0.72 \%{ }^{23} \mathrm{U}\right)$ and of $3 \%, 20 \%$, and $90 \%{ }^{235} \mathrm{U} / \mathrm{U}$ enrichments obtained with a shielded 3 in. $\times 3$ in. Nal detector. 